\section{OP0188 INTEGRATED SAFETY SUMMARY OF THE NOVEL, INTRA-ARTICULAR AGENT LORECIVIVINT (SM04690), A CLK/DYRK1A INHIBITOR THAT MODULATES THE WNT PATHWAY, IN SUBJECTS WITH KNEE OSTEOARTHRITIS}

I. Simsek ${ }^{1}$, C. Swearingen ${ }^{1}$, S. Kennedy ${ }^{1}$, J. Tambiah ${ }^{1}$, C. Damatarca ${ }^{1}$, Y. Yazici', N. Lane ${ }^{2}$, M. Hochberg ${ }^{3} .{ }^{1}$ Samumed, LLC, San Diego, United States of America; ${ }^{2}$ University of California, Davis, Davis, United States of America; ${ }^{3}$ University of Maryland, Baltimore, United States of America

Background: Concerns over the safety of available osteoarthritis (OA) treatments have led to revision of treatment guidelines and highlight the need for new therapies. Lorecivivint (LOR; SM04690) is an intra-articular (IA), small-molecule CLK/DYRK1A inhibitor that modulates the Wnt pathway and is in development as a potential disease-modifying treatment for knee $\mathrm{OA}^{1,{ }^{1,2}}$

Objectives: To evaluate pooled early-phase LOR clinical data for safety, including bone health-related adverse events (AEs).

Methods: Safety data were pooled from 3 randomized controlled trials (one Phase 1, two Phase 2) evaluating 4 doses $(0.03 \mathrm{mg}, 0.07 \mathrm{mg}, 0.15 \mathrm{mg}$, $0.23 \mathrm{mg}$ ) of a single IA injection of LOR in subjects with moderately to severely symptomatic knee OA. Two trials (NCT02095548; NCT03122860) evaluated subjects for 24 weeks and one trial (NCT02536833) for 52 weeks. AEs, serious AEs (SAEs), and bone health AEs were categorized by Medical Dictionary for Regulatory Activities (MedDRA) classification. Incidences of AEs and SAEs were compared between the combined LOR-treated group (subjects who received any dose of LOR) and a control group (subjects not treated with LOR).

Results: This analysis includes 848 LOR-treated and 360 control subjects. The incidence of AEs was similar in LOR-treated (350/848 [41.3\%]) and control subjects $(138 / 360[38.3 \%])$. Incidence of SAEs was $20 / 848(2.4 \%)$ in LOR-treated and $4 / 360(1.1 \%)$ in control subjects. The most commonly reported AE in LORtreated subjects was arthralgia (treated $7.6 \%$, control $7.2 \%$ ) and was the only AE reported at $>5 \%$ in either group (Fig. 1). Target-knee arthralgia was the most common joint-specific AE (treated 6.5\%, control 5.3\%) (Fig. 2). No AEs in other joints exceeded an incidence of $2 \%$ in either group. In all categories, individual AEs were reported at similar rates between groups and no SAEs were deemed related to LOR by investigators.

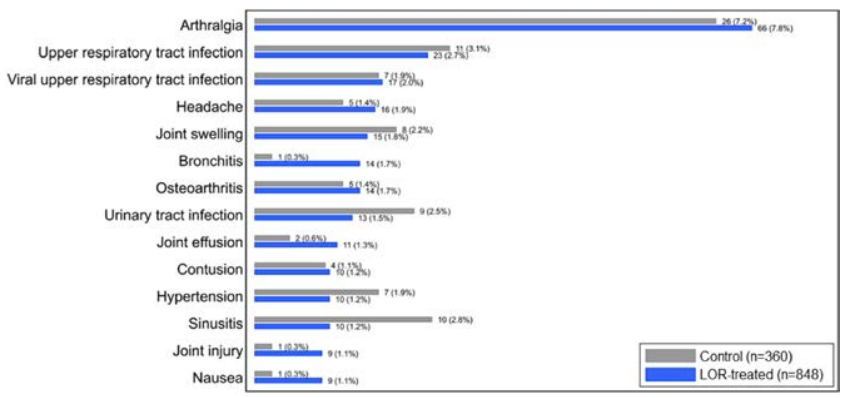

Figure 1. Adverse event summary for events occurring in at least $1 \%$ of the treated population $(\mathrm{N}=1208)$.

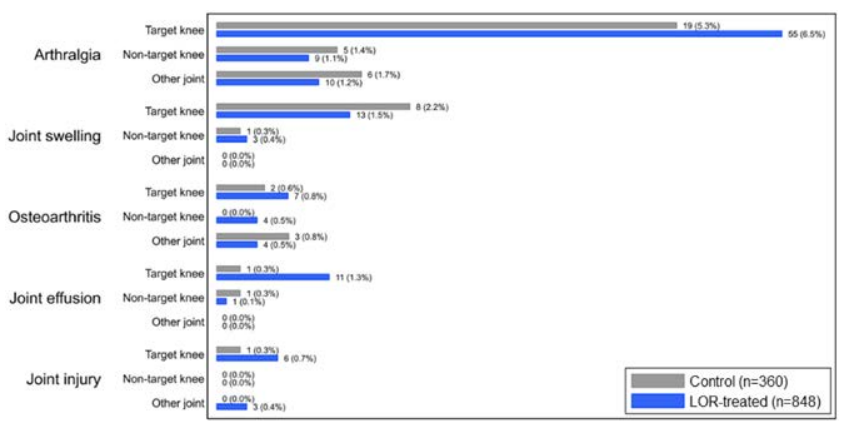

Figure 2. Joint-specific adverse event summary, subcategorized by affected joint, for events occurring in at least $1 \%$ of the treated population $(\mathrm{N}=1208)$.

There were 16 bone health-related AEs in 9/848 (1.1\%) LOR-treated and 3/360 $(0.8 \%)$ control subjects. Of the bone health AEs, 2 were osteopenia/osteoporosis in 2 LOR-treated postmenopausal women and 14 were fractures in 10 subjects (7 LOR-treated, 3 control). All fractures (3 patellar [1 target knee, 2 non-target knee], 3 vertebral, 2 foot, 2 wrist, 2 rib, 1 fibula, 1 hand) were adjudicated and determined to be caused by trauma; all healed uneventfully within the expected time frame.

Conclusion: In exposure to date of 848 subjects, IA LOR for the treatment of knee OA appeared to be safe and well tolerated. These data support the continued evaluation of LOR as a potential treatment for knee OA.

References:

[1] Deshmukh V, et al. Osteoarthritis Cartilage. 2017

[2] Deshmukh V, et al. Osteoarthritis Cartilage. 2019.

Disclosure of Interests: Ismail Simsek Shareholder of: Samumed, LLC Employee of: Samumed, LLC, Christopher Swearingen Shareholder of: Samumed, LLC, Employee of: Samumed, LLC, Sarah Kennedy Shareholder of: Samumed, LLC, Employee of: Samumed, LLC, Jeyanesh Tambiah Shareholder of: Samumed, LLC, Employee of: Samumed, LLC, Cristina Damatarca Shareholder of: Samumed, LLC, Employee of: Samumed, LLC, Yusuf Yazici Shareholder of: Samumed, LLC, Grant/research support from: Bristol-Myers Squibb, Celgene, and Genentech, Consultant of: Celgene and Sanofi, Employee of: Samumed, LLC, Nancy Lane Consultant of: Samumed, LLC, Marc Hochberg: None declared

DOI: 10.1136/annrheumdis-2020-eular.6635

\begin{tabular}{|l|l}
\hline OP0189 & ASSESSMENT OF CARTILAGE DEGRADATION AND \\
PROTECTIVE MARKERS IN SYNOVIAL FLUID FROM \\
OSTEOARTHRITIS PATIENTS BEFORE AND AFTER \\
CYCLES OF INTRA-ARTICULAR INJECTIONS WITH \\
SPRIFERMIN
\end{tabular}

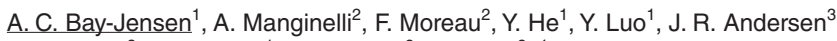
A. R. Bihlet ${ }^{3}$, M. Karsdal ${ }^{1}$, H. Gühring ${ }^{2}$, C. Ladel ${ }^{2} .{ }^{1}$ Nordic Bioscience, Immunoscience, Herlev, Denmark; ${ }^{2}$ Merck KGaA, Darmstadt, Germany; ${ }^{3}$ Nordic Bioscience, Clinical development, Herlev, Denmark

Background: It is challenging to monitor treatment effects after intra-articular (IA) injection with tissue modifying drugs. Assessment of biomarker levels in synovial fluid may be one solution to the challenge. Sprifermin is a truncated form of fibroblast growth factor (FGF) 18 known to induce chondrocyte proliferation and type II collagen formation [1,2]. Data from preclinical investigations show that cartilage formation happens in different phases after therapy with sprifermin, starting with a phase of cartilage degradation during the induction of proliferation of chondrocytes followed by a phase of cartilage formation/production of extracellular matrix.

Objectives: The aim was to investigate the effect of IA administrated sprifermin on cartilage turnover activity as compared to placebo in the injected joint by measurement of markers using longitudinal synovial fluid samples of patients participating in the FORWARD study.

Methods: Each included patient had baseline and at least one FU sample available. Synovial fluid (SF) from participants receiving injections at three consecutive weeks in six month intervals through to week (wk) 80 (fig.A) available from the phase II clinical trial evaluating the efficacy and safety of intraarticularly delivered sprifermin [3] were selected for the investigations. Biochemical markers were measured in available SF samples of the placebo (containing saline IA, $\mathrm{n}=38$ ) and the highest sprifermin dose group (100 mcg/IAx4, $\mathrm{n}=59$ ). Samples were pretreated with ultrasound and centrifugation to decrease viscosity. Markers measured were PRO-C2 (type II collagen formation), huARGS (aggrecan degradation), and FBN-C (fibronectin). Markers are technically validated for synovial fluid measurement. Data were individually normalized to baseline to investigate the median proportional change over time.

Results: Baseline mean (SD) levels of the markers in SF at BL were: PRO-C2, 21.4 (13.6) ng/mL, huARGS, 1117 (516) pM and FBN-C, 2556 (1959) ng/mL. PRO-C2 was initially decreased (from BL to wk 2) after injection with sprifermin; however, the level was increased at the beginning of each new injection cycle followed by a decrease after injection of sprifermin (Fig.B). Overall synovial PRO$\mathrm{C} 2$ levels increased over time in therapy with sprifermin, while no change was observed for the placebo arm. huARGS showed a similar pattern as PRO-C2 - there was an overall increase in ARGS over time in the sprifermin group (fig.C). Interestingly ARGS continuously decreased over time in the placebo group. FBN-C is continuously increased after injection's cycles, whereas no effect was seen in the placebo group (fig.D)

Conclusion: Confirmatory of the preclinical investigations a biphasic response on cartilage turnover after injection with sprifermin was observed. Biochemical indications of cartilage formation and chondrocyte proliferation was only modulated in the sprifermin group, and cartilage degradation (ARGS) was temporal induced and reduced by sprifermin and placebo injections, respectively.

\section{References:}

[1] Gigout A, et al. "Sprifermin (rhFGF18) enables proliferation of chondrocytes producing a hyaline cartilage matrix". Osteoarthr Cartil. 2017;25. 